\title{
Celulitis orbitaria neonatal por Staphylococcus aureus meticilinorresistente de la comunidad
}

\author{
Staphylococcus aureus methicillin-resistant community acquired neonatal \\ orbital cellulitis
}

\begin{abstract}
Dra. M. Guadalupe Péreza , Dra. Graciela Castro ${ }^{b}$,Dra. Celeste Mansillac , Dra. Carina Kaldzielskib, Dra. Gisela Salas ${ }^{b}$ Dra. María Teresa Rosanova $a^{a}$ Dra. Griselda Berberian ${ }^{a}$
\end{abstract}

\section{RESUMEN}

La celulitis orbitaria es una patología del niño mayor y raramente compromete al período neonatal. Staphylococcus aureus (SA) es el principal agente etiológico relacionado. El diagnóstico precoz y el tratamiento adecuado mejoran el pronóstico. Se presentan tres recién nacidos con celulitis orbitaria por SA meticilinorresistente de la comunidad (SAMR-CO).

Palabras clave: SAMR-CO, celulitis orbitaria, neonatos, absceso orbitario.

\section{SUMMARY}

Orbital cellulitis typically occurs in older children, but it can occasionally affect infants and neonates. Staphylococcus aureus is the main pathogen isolated. Outcome depends on an adequate initial approach.

We report three neonates with orbital cellulitis caused by community-associated MRSA.

Key words: MRSA-CA, orbital cellulitis, newborn, orbital abscess.

http:/ /dx.doi.org/10.5546/aap.2013.e50

\section{INTRODUCCIÓN}

Staphylococcus aureus (SA) causa con frecuencia infecciones en pediatría. Últimamente se registra un aumento en la incidencia en niños mayores y en el período neonatal. ${ }^{1}$ Las cepas de Staphylococcus aureus meticilinorresistente proveniente de la comunidad (SAMR-CO) son las prevalentes actualmente. ${ }^{2}$

Presentamos tres neonatos internados en la unidad neonatal del Hospital Nacional de Pediatría "Prof. Dr. Juan P. Garrahan entre el 2009 y 2011, con celulitis y absceso orbitario por SAMR-CO.

a. Servicio de Control Epidemiológico e Infectología.

b. Servicio de Cuidados neonatales.

c. Servicio de Oftalmología.

Hospital Nacional de Pediatría "Prof. Dr. Juan P. Garrahan".

\section{Correspondencia:}

Dra. M. Guadalupe Pérez:

guaperez@hotmail.com

Conflicto de intereses: Ninguno que declarar.

Recibido: 28-7-11

Aceptado: 19-10-11

\section{Caso 1}

Paciente de 13 días de vida que consultó por tumefacción del ojo izquierdo de 48 h de evolución. Se internó con diagnóstico de sepsis y celulitis orbitaria.

$\mathrm{Al}$ ingreso, el niño se encontraba en regular estado general, con eritema periorbitario y proptosis izquierda (Figura 1).

El hemograma presentó 8500 leucocitos por $\mathrm{mm}^{3}$ (72\% neutrófilos), hemoglobina $10,1 \mathrm{~g} / 1, \mathrm{y}$ plaquetas $130 \times 10^{9} / 1$. Se realizaron hemocultivos y punción lumbar. En la evaluación oftalmológica se informó proptosis con fondo de ojo normal. La tomografía axial computada (TAC) de órbita al ingreso evidenció absceso orbitario y subperióstico izquierdo con desplazamiento del globo ocular y etmoiditis (Figura 2. El número 1 señala el absceso orbitario y el 2 el compromiso de las partes blandas periorbitarias).

Recibió inicialmente cefotaxima endovenosa. A las 48 h se informó desarrollo en hemocultivos de SAMR-CO (resistente a meticilina, sensible a vancomicina, clindamicina, teicoplanina, eritromicina, trimetroprima/sulfametoxazol y

FIgURA 1. Paciente con eritema periorbitario y proptosis.

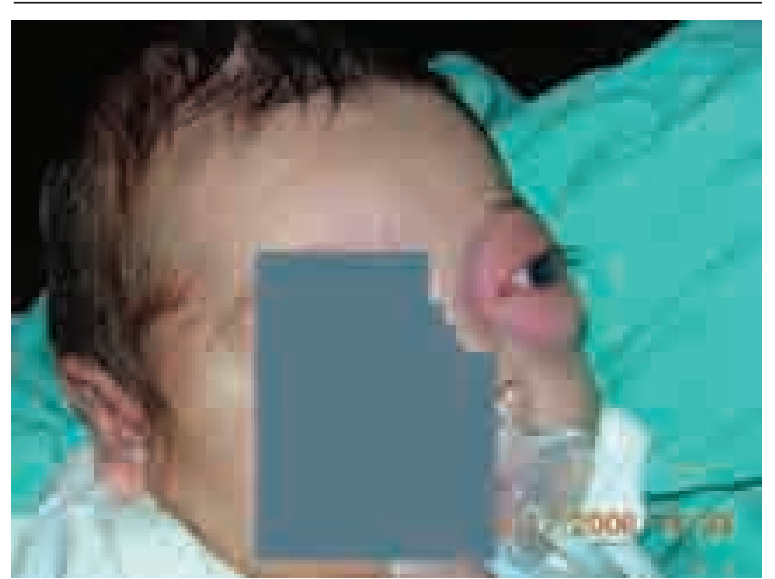


rifampicina), por lo que se indicó vancomicina y rifampicina. Evolucionó favorablemente con este tratamiento, se negativizaron los hemocultivos, sin necesidad de drenaje quirúrgico. A las 3 semanas se realizó una nueva TAC en la que se evidenció la resolución del absceso. Completó 3 semanas de tratamiento endovenoso y luego 5 semanas por vía oral con trimetroprima/sulfametoxazol.

\section{Caso 2}

Paciente de 22 días de vida; sin antecedentes patológicos de importancia. Se internó con diagnóstico de fiebre sin foco aparente de infección. Se realizó un hemograma que evidenciaba 18400 leucocitos por $\mathrm{mm}^{3}$ (51\% neutrófilos), hemoglobina $13,3 \mathrm{~g} / 1$ y plaquetas de $437 \times 10^{9} / 1$. Se tomaron muestras para hemocultivos y urocultivo. Se realizó punción lumbar, que mostró un citoquímico de líquido cefalorraquídeo (LCR) normal. Se inició tratamiento endovenoso con ampicilina y gentamicina.

A las 24 h de internación, persistía febril y comenzó con eritema y edema periorbitario del ojo derecho. Se realizó una TAC que mostró heterogeneidad de la grasa intraconal en contacto con el músculo recto interno. Se modificó el tratamiento a cefotaxima y clindamicina. Los hemocultivos fueron positivos para SAMR-CO (resistente a meticilina, sensible a vancomicina, clindamicina, teicoplanina, eritromicina, trimetroprima/sulfametoxazol y rifampicina). Continuó tratamiento con vancomicina y rifampicina. El paciente evolucionó favorablemente al cuarto día del tratamiento. No requirió cirugía. Fue tratado durante 3 semanas por vía parenteral y luego continuó por

FIGURA 2. TAC: Abceso orbitario (1) y compromiso de partes blandas periorbitarias (2)

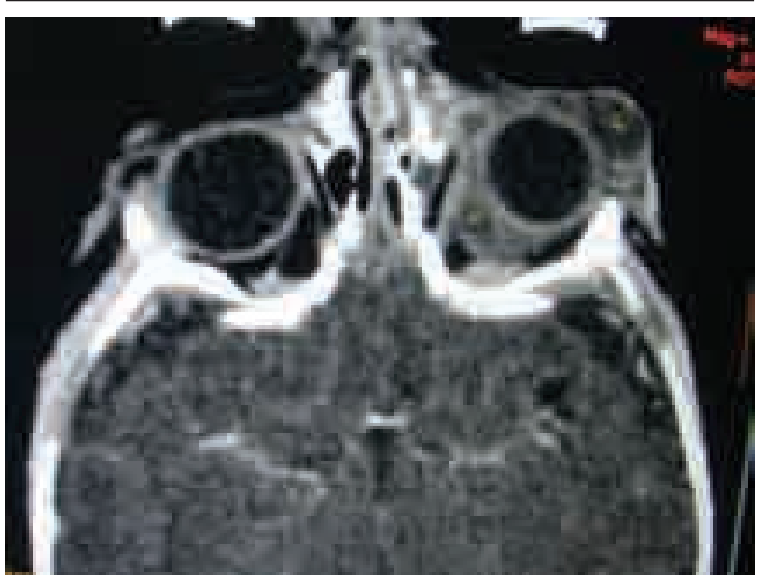

3 semanas vía oral con trimetroprima/sulfametozaxol y rifampicina. Se realizó una TAC al mes de tratamiento que evidenció la resolución completa de la infección.

\section{Caso 3}

Se internó un niño de 4 días de vida con conjuntivitis y tumefacción del ojo izquierdo. Con diagnóstico de sepsis neonatal recibió ampicilina y gentamicina. La madre refería forunculosis en miembros inferiores.

Debido a la progresión de la tumefacción orbitaria y al agregado de convulsiones fue derivado al décimo día de vida al hospital para estudio. El niño ingresó en mal estado general, con convulsiones y eritema y proptosis grave izquierda. $\mathrm{Al}$ ingreso requirió asistencia respiratoria mecánica. Comenzó tratamiento con vancomicina y meropenem. En la TAC de órbita y cerebro se observaba compromiso periorbitario y un absceso orbitario izquierdo con desplazamiento del globo ocular, etmoiditis y múltiples imágenes compatibles con isquemia y abscesos cerebrales. El citoquímico del LCR fue patológico. El LCR y los hemocultivos del ingreso fueron positivos para SAMR-CO con la misma sensibilidad que los casos anteriores. Continuó el tratamiento con vancomicina y rifampicina. Requirió drenaje quirúrgico del absceso orbitario, con cultivo positivo para SAMR-CO. Evolucionó con neumonía bullosa e hidrocefalia aguda. Recibió 6 semanas de vancomicina y rifampicina, y luego trimetroprima/sulfometoxazol y rifampicina. Al egreso, el niño presentó secuelas neurológicas graves con cuadriparesia espástica.

\section{DISCUSIÓN}

La celulitis orbitaria es rara en niños y más aún en el período neonatal. En neonatos se asocia con dacriocistitis, sinusitis etmoidal o tumores de órbita. Los senos etmoidales se encuentran desarrollados al nacer, por lo que puede hallar etmoiditis incluso en niños prematuros. ${ }^{3}$

Hasta la fecha hay descriptos escasos casos de celulitis orbitaria por SAMR-CO en recién nacidos. ${ }^{4-7}$ Debe sospecharse el compromiso orbitario ante el eritema, tumefacción o dolor periorbitario. Al examen oftalmológico, la presencia de proptosis, quemosis y la disminución de los movimientos oculares indican afectación orbitaria. ${ }^{4}$ Para el diagnóstico es necesario realizar una TAC con contraste de la órbita para identificar el compromiso retroorbitario..$^{5}$ Según la TAC, se distinguen en 5 estadios: I: edema preseptal; II: absceso subperióstico; III: celulitis orbitaria; IV: absce- 
so orbitario; V: cualquiera de los anteriores más trombosis de la vena oftálmica o del seno cavernoso. ${ }^{6}$ Los pacientes presentados corresponden a los grados IV, III y V, respectivamente. En el período neonatal, suele ser necesaria la resonancia magnética nuclear (RMN) para evaluar los tejidos blandos adyacentes. ${ }^{8}$

Los hemocultivos son frecuentemente negativos en la celulitis orbitaria. En neonatos, la incidencia de bacteriemia es del 14-20\%. ${ }^{9}$ El mayor rédito microbiológico se obtiene del cultivo del absceso cuando es drenado. En la serie de niños con absceso orbitario presentada por McKinley y cols., todos tuvieron aislamiento microbiológico en el material de drenaje de la lesión. ${ }^{8}$ En el caso de los pacientes anteriormente presentados todos tuvieron sepsis y hemocultivos positivos. El caso 3 tuvo además SAMR-CO en el cultivo del absceso.

En cuanto a los agentes etiológicos aislados en la celulitis orbitaria, Staphylococcus aureus es el más frecuente (60-70\%). ${ }^{10}$ Luego de la introducción de la vacuna conjugada para Haemophilus influenzae $b$ y la disminución de los casos de infección invasiva por este patógeno, Streptococcus pneumoniae, Streptococcus pyogenes y Staphylococcus aureus son las bacterias prevalentes en niños con celulitis orbitaria. ${ }^{9}$

El tratamiento de la celulitis orbitaria constituye una "urgencia infectológica". Requiere intervención adecuada desde el inicio, ya que de ello depende el pronóstico del paciente. El tratamiento es con antibióticos sistémicos en todos los casos y quirúrgico, dependiendo de cada situación. El tratamiento empírico debe estar dirigido contra Staphylococcus aureus, Streptococcus pneumoniae y Streptococcus pyogenes. ${ }^{11}$ El drenaje quirúrgico está indicado cuando existe compromiso del nervio óptico, proptosis con disminución de los movimientos oculares, abscesos que comprimen estructuras nerviosas y en aquellos casos refractarios al tratamiento antibiótico. ${ }^{5,8}$ La celulitis orbitaria con etmoiditis en un recién nacido es siempre una infección grave, con complicaciones igualmente graves. ${ }^{7}$ El tercer paciente presentado fue el más grave de todos ellos, probablemente por la edad temprana de presentación y el retraso del tratamiento adecuado. Sólo el tercer paciente requirió drenaje quirúrgico, el resto curaron con tratamiento antibiótico exclusivo.

Desde el año 2000 existen descripciones aisladas y brotes en nursery de infecciones por SAMR$\mathrm{CO}$ en recién nacidos. ${ }^{1}$ En la unidad de cuidados neonatales del Hospital de Pediatría "Prof. Dr.
Juan P. Garrahan", entre los años 2006-2011, se internaron 20 neonatos con infecciones por SA. El $70 \%$ fueron SAMR-CO (14 pacientes). Todos tuvieron compromiso de la piel o partes blandas. ${ }^{12}$ SAMR-CO se describe como un agente etiológico cada vez más frecuente de infecciones oculares: celulitis preseptal, absceso palpebral, celulitis orbitaria, endoftalmitis y queratitis. ${ }^{13}$ Las descripciones de celulitis con absceso orbitario por SAMR-CO en neonatos sin antecedentes son escasas hasta la fecha. ${ }^{5}$ La gravedad del cuadro clínico y la intensa morbilidad demandan un tratamiento adecuado y eficaz.

Por todo lo previamente mencionado, siendo SA el principal agente etiológico, y con el aumento en nuestro medio de las infecciones por SAMR-CO, la vancomicina debería incorporarse al tratamiento empírico. Estas recomendaciones coinciden con lo propuesto por la Sociedad Estadounidense de Infectología en el año 2010, que sugiere el uso de vancomicina para la sepsis por SAMR-CO en el período neonatal. Se reserva el uso de clindamicina y linezolide para infecciones leves o moderadas. ${ }^{14}$

\section{CONCLUSIÓN}

Presentamos tres recién nacidos con celulitis orbitaria y etmoiditis con identificación de SAMR$\mathrm{CO}$. Esta infección es rara y requiere un diagnóstico precoz y manejo multidisciplinario. La TAC o RMN son indispensables en la evaluación inicial del compromiso ocular.

Teniendo en cuenta que las infecciones por SAMR-CO en la población neonatal han aumentado en los últimos años, debe considerarse el agregado de vancomicina al tratamiento empírico en pacientes con sospecha de infecciones por SAMR-CO.

\section{BIBLIOGRAFÍA}

1. Fortunov R, Hulten $\mathrm{K}$, Hammerman W. Community-acquired Staphylococcus aureus infections in term and near term previously healthy neonates. Pediatrics 2006; 118: 874881.

2. Paganini HR, Della Latta P, Soto A, Casimir L, et al. Bacteriemias por Staphylococcus aureus adquiridas en la comunidad: 17 años de experiencia en niños de la Argentina. Arch Argent Pediatr 2010; 108(4):311-7.

3. Soon V. Pediatric subperiosteal orbital abscess secondary to acute sinusitis: a 5 year review. Am J Otolaryngology 2011; 32(1):62-8.

4. Anari S, Karagama Y, Fulton B, Wilson JA. Neonatal disseminated methicillin-resistant Staphylococcus aureus presenting as orbital cellulitis. J Laryngology Otology 2005; 119:64-7.

5. McCarty M, Myer C. Orbital cellulitis in children. Pediatr Infect Dis J 2007; 26(3):276-7. 
6. Ho CF, Huang YC, Wang $\mathrm{CJ}$, $\mathrm{Chiu} \mathrm{CH}$, et al. Clinical analysis of computed tomography-staged orbital cellulitis in children. J Microbiol Inmunol Infect 2007; 40(6):518-24.

7. Swift AC, Charlton G. Sinusitis and the acute orbit in children. J Laryngol Otol 1990; 104: 213-6.

8. McKinley S, Yen M, Miller A, Yen K. Microbiology of pediatric orbital cellulitis. Am J Ophthalmology 2007; 144:497-501.

9. Molarte $A B$, Isenberg SJ. Periorbital celullitis in infancy. Ped Infect Dis J 1990; 9(8):603.

10. Skedros DG, Haddad J, Bluestone CD, Curtin HD. Subperiosteal abscess in children: diagnosis, microbiology and management. Laryngoscope 1993; 103:28.

11. Seltz LB, Smith J, Durairaj VD, Enzenauer R. Microbiology and antibiotic management of orbital cellulitis. Pediatrics 2011; 127(3):e566-72.
12. Berberian G, Castro G, Monaco A. Community-acquired Methicillin-resistant Staphylococcus aureus infection in previously healthy neonates. 14th International Congress on Infectious Diseases. Florida. Miami, Estados Unidos. 9 al 12 Marzo 2010. Poster 76.003.

13. Goldstein S, Shelsta H. Community-acquired methicillin-resistant Staphylococcus aureus periorbital cellulitis: a problem here to stay. Ophthal Plast Reconstr Surg 2009; 25(1):77-8.

14. Liu C, Bayer A, CosgroveS, Daum RS, et al. Clinical practice guidelines by the Infectious Diseases Society of America for the treatment of methicilin-resistant Staphylococcus aureus infections in adults and children. Clin Infect Dis 2011; 52(1):1-38. 